\title{
Dealing with Uncertainties in MCDA
}

DOI:

10.1007/978-1-4939-3094-4_12

\section{Document Version}

Accepted author manuscript

Link to publication record in Manchester Research Explorer

\section{Citation for published version (APA):}

Stewart, T., \& Durbach, I. (2016). Dealing with Uncertainties in MCDA. In S. Greco, M. Ehrgott, \& J. Figueira (Eds.), Multiple Criteria Decision Analysis: Satte of the Art Surveys (pp. 467-496). (International Series in Operations Research \& Management Science; Vol. 233). Springer Nature. https://doi.org/10.1007/978-1-49393094-4_12

\section{Published in:}

Multiple Criteria Decision Analysis: Satte of the Art Surveys

\section{Citing this paper}

Please note that where the full-text provided on Manchester Research Explorer is the Author Accepted Manuscript or Proof version this may differ from the final Published version. If citing, it is advised that you check and use the publisher's definitive version.

\section{General rights}

Copyright and moral rights for the publications made accessible in the Research Explorer are retained by the authors and/or other copyright owners and it is a condition of accessing publications that users recognise and abide by the legal requirements associated with these rights.

\section{Takedown policy}

If you believe that this document breaches copyright please refer to the University of Manchester's Takedown Procedures [http://man.ac.uk/04Y6Bo] or contact uml.scholarlycommunications@manchester.ac.uk providing relevant details, so we can investigate your claim.

\section{OPEN ACCESS}


Final Preprint of paper published as Chapter 12, pp. 467-496, in S. Greco et al. (eds.), Multiple Criteria Decision Analysis: State of the Art Surveys, International Series in Operations Research \& Management Science 233, 2016

\title{
Dealing with Uncertainties in MCDA
}

\author{
Theodor Stewart \\ Department of Statistical Sciences \\ University of Cape Town \\ Rondebosch, 7701, South Africa \\ Ian Durbach \\ Department of Statistical Sciences \\ University of Cape Town \\ Rondebosch, 7701, South Africa \\ and \\ Research Center \\ African Institute for Mathematical Sciences \\ 6-8 Melrose Road, Muizenberg, 7945, South Africa
}

\section{What is Uncertainty?}

The term uncertainty can have many different meanings. The Chambers Dictionary (1998 edition) defines "uncertain" as not definitely known or decided; subject to doubt or question. [60] quote six different definitions for "uncertainty" from Webster's Dictionary. In the context of practical applications in multicriteria decision analysis, however, the definition given by [108] would appear to be particularly appropriate. With minor editing, this is as follows:

Uncertainty implies that in a certain situation a person does not possess the information which quantitatively and qualitatively is appropriate to describe, prescribe or predict deterministically and numerically a system, its behaviour or other characteristics.

At a most fundamental level, uncertainty relates to a state of the human mind, i.e. lack of complete knowledge about something. Many writers also use the term "risk", although the definition of the term varies widely. Some earlier work tended to apply the term "risk" to situations in which probabilities on outcomes are (to a large extent) known objectively (cf. [38], p. 389, and [75] for some reference to this view). More recently, the concept of risk has come to refer primarily to the desirability or otherwise of uncertain outcomes, in addition to simple lack of knowledge. Thus, for example, [33] refers to risk as "a chance 
of something bad happening", and in fact separates uncertainty (alternatives with several possible outcome values) from the fundamental concept of risk as a bad outcome. [86] state that "judgements about riskiness depend on both the probability and the magnitude of adverse effects" (our emphasis), while [53] also discuss the psychological aspects of establishing a preference order on risks.

For the most part in this chapter, we shall make use of the value-neutral term "uncertainty", referring to "risk" only when direct preference orderings of the uncertainty per se are relevant (for example, in Section 5). It is interesting to note in passing that while the thrust of the present discussion is to give consideration to the effects of uncertainty on MCDA, there has also been work on applying multicriteria concepts to the measurement of risk for other purposes, as for example in credit risk assessment ([27], who make use of a rough sets approach).

A number of authors (e.g. [34], [108]) have attempted to categorize types or sources of uncertainty in the context of decision making. [34], for example, identifies no less than 10 different sources of uncertainty which may arise in model building for decision aid, which he classifies into three groups referring broadly to uncertainties in the modelling (or problem structuring) process, in the use of models for exploring trends and options, and in interpreting results. The common theme underlying such categorizations, as well as those of other authors, such as [36] and [68], is the need at very least distinguish between internal uncertainty, relating to the process of problem structuring and analysis, and external uncertainty, regarding the nature of the environment and thereby the consequences of a particular course of action which may be outside of the control of the decision maker. Let us briefly examine each of these broad categories of uncertainty.

Internal uncertainty This refers to both the structure of the model adopted and the judgmental inputs required by those models, and can take on many forms, some of which are resolvable and others which are not. Resolvable uncertainties relate to imprecision or ambiguity of meaning - for example, what exactly may be meant by a criterion such as "quality of life"? Less easily resolvable problems may arise when different stakeholders generate different sets of criteria which are not easily reconciled; or perceive alternatives in such different ways that they differ fundamentally on how they contribute to the same criterion.

Imprecisions in human judgments, whether these relate to specifications of preferences or values (for example importance weights in many models), or to assessments of consequences of actions, have under certain circumstances been modelled by fuzzy set (see, for example, Chapters 4 and 5 of [60]) and related approaches (such as the use of rough sets as described by [41, 40, 42]. From the point of view of practical decision aid, such models of imprecision add complexity to an already complex process, and the result may often be a loss of transparency to the decision maker, contrary to the ethos of MCDA. For this reason, the view espoused here is that internal uncertainties should ideally be resolved as far as 
is possible by better structuring of the problem (cf. [11], Chapter 3) and/or by appropriate sensitivity and robustness analysis where not resolvable, which will further be discussed in Section 2. The evidential reasoning (ER) approach described by [101], to which we shall refer again at the end of Section 3, does provide a more formal model for integrating imprecise preference information that cannot fully be resolved.

External uncertainty This refers to lack of knowledge about the consequences of a particular choice. [36] and [34] both recognize a further distinction between uncertainty about the environment and uncertainty about related decision areas, as described below.

- Uncertainty about the environment represents concern about issues outside the control of the decision maker. Such uncertainty may be a consequence of a lack of understanding or knowledge (in this sense it is similar to uncertainty about related decision areas) or it may derive from the randomness inherent in processes (for example the chance of equipment failure, or the level of the stock market). For example, the success of an investment in new production facilities may rest on the size of the potential market, which may depend in part on the price at which the good will be sold, which itself depends on factors such as the cost of raw materials and labour costs. A decision about whether or not to invest in the new facilities must take all of these factors into account. This kind of uncertainty may be best handled by responses of a technical nature such as market research, or forecasting.

- Uncertainty about related decision areas reflects concern about how the decision under consideration relates to other, interconnected decisions. For example, suppose a company which supplies components to computer manufacturers is looking to invest in a management information system. They would like their system to be able to communicate directly with that of their principal customers; however, at least one of these customers may be planning to install a new system in the near future. This customer's decision could preclude certain of the options open to the supplier and would certainly have an impact on the attractiveness of options. The appropriate response to uncertainty of this kind may be to expand the decision area to incorporate interconnected decisions, or possibly to collaborate or negotiate with other decision makers.

Under many circumstances, both internal and external uncertainties can be treated in much the same manner, for example by appropriate sensitivity analyses post hoc. In other words, the approach might be to make use of a crisp deterministic MCDA methodology, and to subject the results and conclusions to extensive sensitivity studies. Indeed, we would assert that such sensitivity studies should routinely be part of any MCDA application, and some approaches are discussed in Section 2. 
Where uncertainties are of sufficient magnitude and importance to be modelled explicitly as part of the MCDA methodology, however, the modelling approaches for internal and external uncertainties may often become qualitatively different in nature. It seems, therefore, that the treatment of the two types of uncertainty should preferably be discussed in separate papers or chapters. In order to provide focus for the present paper, our attention will be focussed primarily, apart from Section 2, on consideration of the external uncertainties as defined above. Without in any way minimizing the importance of dealing with internal uncertainties, our choice of the problem of external uncertainties as the theme for this chapter is in part due to the present authors' practical experience, which suggests that it is the external uncertainties which are often of sufficient magnitude and importance to require more explicit modelling. The present chapter complements in many ways the survey paper by [31] which does include more on internal uncertainties and the behavioural models of uncertainty and risk perception. (It should perhaps be acknowledged that there is also some inevitable overlap between [31] and the current chapter, but the thrusts are still distinct.)

Admittedly, the boundary between external uncertainty and imprecision is, well, fuzzy! To this extent, some of the material in this chapter is appropriate to internal uncertainties as well, while some methods formulated to deal with human imprecision might equally well be useful in dealing with external uncertainties. We leave it to the reader to decide where this may be true. We do not attempt here a comprehensive review of literature related primarily to internal uncertainties, but the interested reader may wish to consult some of the following references:

- Fuzzy set approaches: [60]; [22, 21]; [103]; some discussion may also be found in [31];

- Rough set approaches: [41, 40, 42, 43].

Our approach is pragmatic in intention, motivated by practical needs of realworld decision analysis. In particular, the fundamental philosophical point of departure is a belief in the over-riding need for transparency in any MCDA: it is vitally and critically important that any approaches to MCDA are fully understandable to all participants in the process. Elegant mathematical models which are inaccessible to such participants are of very little practical value.

Within the context of the opening discussion, let us now define a notational framework within which to consider MCDA under uncertainty (primarily "external uncertainty" as defined earlier). Let $X$ be the set of actions or decision alternatives. When there is no uncertainty about the outcomes, there exists a one-to-one correspondence between elements of $X$ and consequences in terms of the criteria, and $X$ may written as the product space $\prod_{i=1}^{n} X_{i}$, where $X_{i}$ is the set of evaluations with respect to criterion $i$. In other words, any $x \in X$ may be viewed as an $n$-dimensional vector with elements $x_{i} \in X_{i}$, where $x_{i}$ represents the evaluation of $x$ with respect to the criterion $i$. 
Under uncertainty, however, the one-to-one correspondence between actions and evaluations or consequences breaks down. It may be possible to postulate or to conceptualize an ultimate set of consequences $Z_{1}(x), \ldots, Z_{n}(x)$ corresponding to each of the criteria, but at decision time there will still exist many possible values for each $Z_{i}(x)$. For ease of notation, we shall use $\mathbf{Z}(x)$ to indicate the vector of $Z_{i}(x)$ values.

In some cases, it may be possible and useful to structure $Z_{i}(x)$ (or $\mathbf{Z}(x)$ ) in the form $Z_{i}(x, \xi)$ (or $\mathbf{Z}(x, \xi)$ ), where $\xi \in \Xi$ fully characterizes the external conditions, sometimes termed the "states of nature", and $\Xi$ represents the set of all possible states of nature. The assumption is then that once $\xi$ (the state of nature) is established or revealed, then the consequences in terms of each criterion will also be known. We observe, however, that even $\Xi$ might not be fully known or understood at decision time, and that $\Xi$ could possibly depend upon the action $x$ (although, for ease of notation, we shall not show this explicitly).

The question to be addressed in this chapter is that of constructing some form of (possibly partial) preference ordering on $X$, when the consequences are incompletely known or understood in the sense described in the previous paragraph.

As indicated earlier, one approach may be initially to ignore the uncertainty, and to conduct the analysis on the basis of a nominal set of consequences $z_{1}, z_{2}, \ldots, z_{n}$ chosen to be representative of the possible $Z_{i}(x)$, followed by extensive sensitivity analysis which takes into account the range of uncertainty in each $Z_{i}(x)$. Under many circumstances this may be adequate. Care needs to be exercised in undertaking sensitivity analyses, however, as simple "one-ata-time" variations in unknown parameter values may fail to identify effects of higher order interactions. Some of the complications inherent in undertaking properly validated sensitivity analyses, and suggestions as to how these may be addressed, are discussed by [82], [80] and [85]. Section 2 describes some practical approaches for managing such sensitivity studies.

In the remainder of this chapter, the focus will be on situations in which the ranges of uncertainty are too substantial to be handled purely by sensitivity analysis. In Section 3 we discuss the use of probability models and related methods to represent the uncertainties formally, emphasizing particularly the comprehensively axiomatized approach of multiattribute utility theory. The potential for relaxing the needs to specify complete utility functions are addressed in Section 4, which leads naturally to the use of pairwise comparison models for MCDA. In many practical situations, decision maker preferences for various types of risk (magnitude and impact of the uncertainties) may be modelled by defining explicit risk-avoidance criteria, and these are discussed in Section 5. Finally, links between MCDA and scenario planning for dealing with uncertainties are presented in Section 6, before concluding with some general implications for practice. 


\section{Sensitivity analysis and related methods}

For the purposes of this section, we postulate the existence of an "evaluation function" $\Psi(\mathbf{Z}(x, \xi), \phi)$, which indicates a degree of satisfaction associated with the outome of the decision. In this formulation:

- The function $\Psi(\mathbf{Z}(x, \xi), \phi)$ could be a utility, a distance from a desired outcome, etc.;

- The factors $\xi$ and $\phi$ represent respectively the external influences (incompletely known, and outside of the decision makers' control) on consequences of the decision, and the internal uncertainties as to how these consequences should be evaluated in terms of decision maker goals (e.g. importance weights, tradeoffs).

The aim of sensitivity analysis is typically to identify potentially optimal solutions amongst uncertainty ranges in $\xi$ (external) and $\phi$ (internal). Sensitivity analysis is aimed at providing insights into:

1. whether the outcome of the decision model changes as $\xi$ and/or $\phi$ take on different values within the stated bounds. For simplicity of presentation here, we shall assume a choice problematique i.e. the selection of a single preferred alternative;

2. the values of $\xi$ and $\phi$ for which each alternative may be deemed to be the best.

Sensitivity analysis is most appropriately applied when the uncertainties are essentially subjective in nature, i.e. either internal uncertainties $(\phi)$ or situations in which the state is already determined (not subject to future random fluctuations) but still unknown. For ease of presentation we shall denote the combination of subjective uncertainties in state (typically state probabilities) and internal uncertainties by $\psi=(\xi, \phi)$, and assume that there are no other external random influences. In this case, we shall express the evaluation function simply as $\Psi(\mathbf{Z}(x), \psi)$.

If the decision maker has provided a precise specification of elements of $\psi$, sensitivity analysis involves varying $\psi$ away from these specified values and examining the impact on results. This can be done in an ad hoc fashion, although a preferable approach is to use one of the many well-known methods for systematically exploring the space of possible preference parameters (see the review in [51]). Many of the so-called "interactive" or "progressive articulation of preferences" methods (e.g. [91]) may also be useful as tools for sensitivity analysis.

If no precise specification of $\psi$ can be given, alternative forms of sensitivity analysis are provided by inverse-preference and preference disaggregation models. (Interval-based decision models [74] may also be used, but fall outside the scope of the aims of present section.) Inverse preference models typically work by providing information about the volume and types of values for $\psi$ (if any) that would lead to the selection of each alternative. Effectively, instead of asking 'which alternative is best given a particular $\psi$ ?', one asks for example 'what 
ranges of or possible values for $\psi$ would result in a particular alternative being considered the best?'. Partial or total ignorance about possible values for $\psi$ is incorporated through appropriate probability distributions defined over these inputs.

One such inverse-preference method is stochastic multi-criteria acceptability analysis (SMAA). The original SMAA method [65] analysed the combinations of attribute weights (internal uncertainties) that result in each of a set of prospective alternatives being selected when using an additive utility function. Subsequently a number of variants have been developed. These differ in terms of the preference model used and the type of information that is imprecisely known, but are all based upon Monte Carlo simulation from distributions which indicate the extent of the uncertainty in $\psi$. For example, SMAA variants are available for value functions [65, 63], outranking [49], reference point methods $[66,28]$, and prospect theory [64] methods. Several probabilitistic AHP models $[67,7]$ also use Monte Carlo simulation to randomly generate pairwise evaluations from the distributions specified by decision makers, in similar fashion to SMAA.

For illustration, the process described here relates to uncertain importance weight information, but can readily be extended to other subjective uncertainties. SMAA in this context is based on simulating a large number of random weight vectors from a probability distribution defined over the weight space and observing the proportion and distinguishing features of weight vectors which result in each alternative obtaining a particular rank $r$ (usually the "best" rank, $r=1$ ). Other uncertain evaluations, e.g. partial value assessments in value function methods, are also conventionally treated in SMAA using probability distributions, with each simulation run drawing values at random from these distributions. Adapting SMAA models to use other uncertainty formats, however, is generally straightforward [29]. In any case, in order to illustrate the process for uncertain weights, let the set of (randomly generated) weight vectors that result in alternative $a_{i}$ obtaining rank $r$ be denoted by $W_{i}^{r}$. SMAA is based on an analysis of these sets of weights using a number of descriptive measures, the most important of which are:

Acceptability indices The rank- $r$ acceptability index $b_{i}^{r}$ measures the proportion of all simulation runs i.e. weight vectors, that make alternative $a_{i}$ obtain rank $r$. A cumulative form of the acceptability index called the $k$-best ranks acceptability index is defined as $\mathcal{B}_{i}^{k}=\sum_{r=1}^{k} b_{i}^{r}$ and measures the proportion of all weight vectors for which alternative $a_{i}$ appears anywhere in the best $k$ ranks.

Central weight vectors The central weight vector $\mathbf{w}_{i}^{c}$ is defined as the center of gravity of the favourable weight space $W_{i}^{1}$. It gives a concise description of the "typical" preferences supporting the selection of a particular alternative $a_{i}$, and in practice is computed from the empirical (element-wise) averages of all weight vectors supporting the selection of $a_{i}$ as the best alternative. 
Ranges on favourable weights These simply indicate the minima and maxima of the observed favourable weights supporting alternative $a_{i}$.

Preference disaggregation models also aim to provide information on conditions under which one or more alternatives may be preferred to others, particularly with regard to internal uncertainty. These models typically use a set of global preference statements to infer the parameters of a preference model before applying that model to a larger set of alternatives to arrive at a choice or ranking or classification. In the original UTA method (see [52]), the breakpoints of piecewise linear marginal value functions are estimated by a linear program whose main elements are the constraints $U(a)>U(b) \Longleftrightarrow a \succ b$ and $U(a)=U(b) \Longleftrightarrow a \sim b$, along with some technical constraints (e.g. imposing montonicity and a zero-point). Of course, more than one set of value functions may be compatible with the specified global preference statements. The robust ordinal regression approach [45] addresses this issue by providing "necessary" preference relations indicating support from all compatible value functions, and "possible" preference relations indicating support from at least one compatible value function. The necessary and possible rankings are again found using a linear programing formulation, in which a key role is played by the sign of a deviational variable $\epsilon$. An alternative $a$ is "possibly" preferred to $b$; if $\max \epsilon>0$ subject to two constraints $U(a) \geq U(b)$ and $U(a) \geq U(b)+\epsilon$ if $x \succ y$. Then, $a$ is "necessarily" preferred to $b$; if $\max \epsilon \leq 0$ subject to two constraints $U(b) \geq U(a)+\epsilon$ and $U(a) \geq U(b)+\epsilon$ if $x \succ y$.

In addition, preference statements can be in the form of ranking preference differences as well as alternatives, and value functions are not constrained to be piecewise linear. A number of extensions of the basic robust ordinal regression approach have been made to accommodate sorting problems [44], nonadditive functions [4], and outranking methods [46].

This section on sensitivity analysis has focussed on subjective and particularly internal uncertainties. We shall now, for the remainder of the chapter focus on external uncertainties.

\section{Probabilistic Models and Expected Utility}

The most thoroughly axiomatized mathematical treatment of uncertainty is that of probability theory, and possibly extensions such as Dempster-Shafer theory [87]. The application of probability concepts requires the specification of a (multivariate) probability distribution on $\mathbf{Z}(x)$ for each action $x$, so that in effect the decision requires a comparison of probability distributions (sometimes called "lotteries" in this context). Let $\mathbf{P}^{x}(\mathbf{z})$ denote the probability distribution function on $\mathbf{Z}(x)$, i.e.:

$$
\mathbf{P}^{x}(\mathbf{z})=\operatorname{Pr}\left[Z_{1}(x) \leq z_{1}, Z_{2}(x) \leq z_{2}, \ldots, Z_{n}(x) \leq z_{n}\right] .
$$

Define $P_{i}^{x}\left(z_{i}\right)$ as the corresponding marginal probability distribution function for $Z_{i}(x)$. 
Where uncertainties are structured in terms of "states of nature", the probability distributions may be defined on the $\xi$ (rather than on the $\mathbf{Z}(x)$ directly). In some situations, the probability distribution on $\xi$ may be independent of the action which would make the application of probability models much more tractable, but this will not necessarily always be the case.

A possibility at this stage is to construct a deterministic MCDA model based only on expectations, and to subject the results to some form of (possibly interactive) sensitivity analysis, such as described in the previous section, guided by the known distributional properties. Examples of this are in the PROTRADE method described by [38] Chapter 7, dealing with an interactive method for multiobjective mathematical programming problems, and in the stochastic extensions to outranking proposed by [71].

Although simulations reported in [30] suggest that simple expectation models can often return similar results to models taking the full ranges of outcomes into account, this conclusion clearly cannot be generalized to all situations. Multiattribute utility theory (MAUT) extends the concept of expectation to include explicit modelling of risk preferences, i.e. of the magnitudes of dispersion that may occur. MAUT is discussed by Dyer in Chapter dyerchapter of this volume, and also more comprehensively in the now classic texts of [56] and [100]. In essence, MAUT seeks to construct a "utility function" $U(\mathbf{Z})$, such that for any two actions $x$ and $y$ in $X, x \succsim y$ if and only if $\mathrm{E}[U(\mathbf{Z}(x))] \geq \mathrm{E}[U(\mathbf{Z}(y))]$, where expectations are taken with respect to the probability distributions on $\mathbf{Z}(x)$ and on $\mathbf{Z}(y)$ respectively.

Practically, the construction of the global utility function $U(\mathbf{Z})$ starts with the construction of partial or marginal utility functions individually for each attribute, say $u_{i}\left(Z_{i}\right)$, satisfying the expected utility hypothesis for variations in $Z_{i}$ only. The axioms underlying the existence of such marginal utility functions and the methods for their construction are well-known from univariate decision analysis (see, for example, Chapter dyerchapter, or [39], check Chapter 6). It is well-established that these axioms are not descriptively valid, in the sense that decision makers do systematically violate them (see, for example, the various paradoxes described by [55], or in the text of [9]). Attempts have been made to extend the utility models to account for observed behaviour (see, for example, [77] for a review of such extensions in the multicriteria context). Nevertheless, as we have argued elsewhere (e.g.,[11], Section 4.3.1), descriptive failures do not lessen the value of the simpler axiomatically based theory of MAUT as a coherent discipline within which to construct preferences in a simple, transparent and yet defensible manner.

The real challenge relates to the aggregation of the $u_{i}\left(Z_{i}\right)$ into a $U(\mathbf{Z})$ still satisfying the expected utility hypothesis for the multivariate outcomes. The two simplest forms of aggregation are the additive and multiplicative, which we shall now briefly review (although a full description can be found in Chapter dyerchapter. 
Additive aggregation. In this case, we define:

$$
U(\mathbf{Z})=\sum_{i=1}^{n} k_{i} u_{i}\left(Z_{i}\right)
$$

This model is only justifiable if the criteria are additively independent, i.e. if preferences between the multivariate lotteries depend only on the marginal probability distributions. That this is not an entirely trivial assumption may be seen by considering two-dimensional lotteries $(n=2)$ in which there are only two possible outcomes on each criterion, denoted by $z_{i}^{0}$ and $z_{i}^{1}$ for $i=1,2$. Suppose that $z_{i}^{1} \succ z_{i}^{0}$. Then without loss of generality, the partial utility functions can be standardized such that $u_{1}\left(z_{1}^{0}\right)=u_{2}\left(z_{2}^{0}\right)=0$ and $u_{1}\left(z_{1}^{1}\right)=u_{2}\left(z_{2}^{1}\right)=1$. Consider then a choice between two lotteries defined as follows:

- The lottery giving equal chances on $\left(z_{1}^{0} ; z_{2}^{0}\right)$ and $\left(z_{1}^{1} ; z_{2}^{1}\right)$; and

- The lottery giving equal chances on $\left(z_{1}^{0} ; z_{2}^{1}\right)$ and $\left(z_{1}^{1} ; z_{2}^{0}\right)$.

We note that both lotteries give the same marginal distributions on each $Z_{i}$, i.e. equal chances on each of $z_{i}^{0}$ and on $z_{i}^{1}$ for each $i$. It is easily verified that with additive aggregation defined by (1), both of these lotteries yield an expected utility of $\left(k_{1}+k_{2}\right) / 2$. The additive model thus suggests that the decision maker should always be indifferent between these two lotteries. There seems, however, to be no compelling axiomatic reason for forcing indifference between the above two options. Where there is some measure of compensation between the criteria (in the sense that good performance on one can compensate for poorer outcomes on the other), the second option may be preferred as it ensures that one always gets some benefit (a form of multivariate risk aversion). On the other hand, if there is need to ensure equity between the criteria (if they represent benefits to conflicting social groups, for example), then the first lottery (in which loss or gain is always shared equally) may be preferred.

Multiplicative aggregation. Now we define $U(\mathbf{Z})$ such that:

$$
1+k U(\mathbf{Z})=\prod_{i=1}^{n}\left[1+k k_{i} u_{i}\left(Z_{i}\right)\right]
$$

where the multivariate risk aversion $k$ parameter satisfies:

$$
1+k=\prod_{i=1}^{n}\left[1+k k_{i}\right]
$$

Use of the multiplicative model requires that the condition of mutual utility independence be satisfied. A subset of criteria, say $C \subset\{1,2, \ldots, n\}$ is said to be utility independent of its complement $\bar{C}=\{1,2, \ldots, n\} \backslash C$, if preferences for lotteries involving only $Z_{i}$ for $i \in C$ for fixed values of 
$Z_{i}$ for $i \in \bar{C}$ are independent of these fixed values. The criteria are said to be mutually utility independent if every subset of the criteria is utility independent of its complement.

In principle, however, there are no compelling reasons why criteria should necessarily be mutually utility independent, and in fact it can be difficult in practice to verify that the condition holds. Good problem structuring for MCDA would seek to ensure preferential independence of some form between criteria (for example, such that trade-offs between pairs of criteria are independent of outcomes on other criteria), but mutual utility independence is a stronger assumption and more elusive concept than simple preferential independence.

Models based on weaker preference assumptions have been developed, such as the multilinear model given by:

$$
\begin{aligned}
U(\mathbf{Z})=\sum_{i=1}^{n} k_{i} u_{i}\left(Z_{i}\right)+\sum_{i=1}^{n} \sum_{i<j \leq n} k_{i j} u_{i}\left(Z_{i}\right) u_{j}\left(Z_{j}\right) \\
+\ldots+k_{12 \ldots n} u_{1}\left(Z_{1}\right) u_{2}\left(Z_{2}\right) \ldots u_{n}\left(Z_{n}\right)
\end{aligned}
$$

The large number of parameters which have to fitted to decision maker preferences is prohibitive in most real world applications. Even the multiplicative model is far from trivial to apply in practice. Its construction involves the following steps:

- Assessment of the partial utilities $u_{i}\left(Z_{i}\right)$ by standard single attribute lottery procedures.

- Parameter estimation: The multiplicative model includes $n+1$ parameters which have in principle to be estimated, although in the light of (3), only $n$ independent parameters need estimation. Estimates thus require at least $n$ preference statements concerning hypothetical choices to be made by the decision maker. Some of these can be based on deterministic tradeoff assessments, but at least one of the hypothetical choices must involve consideration of preferences between multivariate lotteries.

In exploring the literature, it is difficult to find many reported applications even of the multiplicative model, let alone the multilinear model. Some of the practical complications of properly implementing these models are illustrated by [84] and [104].

Such difficulties of implementation raise the question as to how sensitive the results of analysis may be to the use of the additive model (1) instead of the more theoretically justifiable aggregation models given by (2) or (4). We have seen earlier that situations can be constructed in which the additive model may generate misleading results. But how serious is this in practice? Construction of the additive model requires much less demanding inputs from the decision maker, and it may be that the resultant robustness or stability of the model will compensate for biases introduced by use of the simpler model. 
In [88] a number of simulation studies are reported in which the effects are studied of using the additive aggregation model when "true preferences" follow a multiplicative aggregation model. Details may be found in the cited reference, but in essence it appeared that the errors introduced by using the additive model were generally small for realistic ranges of problem settings. The errors were in any case substantially smaller than those introduced by incorrect modelling of the partial utility functions (such as by over-linearization of the partial functions which appears to be a frequent but erroneous simplification). Related work ([89]) has also demonstrated that more fundamental violations of preferential independence may also introduce substantial errors.

Concerns about the validity of the fundamental axiomatic foundations of utility theory, even for single criterion problems, have led other writers to formulate alternative models to circumvent these. From the standpoint of prescriptive decision aid, a particular concern is that several utility techniques for eliciting the marginal value functions $u_{i}\left(z_{i}\right)$ (e.g. certainty-equivalence and probabilityequivalence methods) assume that the axioms of EUT hold during the elicitation process [16], even though these axioms are known not to be descriptively valid. Utility function assessments based on elicited responses from decision makers who do not follow EUT may thus be systematically biased. Importantly, this concern for the validity of estimated marginal utility functions relates to observed or descriptive behaviour, and is thus independent of any debate around the desirability of the axioms in a normative decision aiding sense. [96] propose an alternative assessment method - the gamble trade-off method - that does not depend on the actual probability values, and is thus robust to the kinds of probability transformations that decision makers often use. These procedures are extended in $[1,15]$ to allow for the assessment of both non-expected utility and probability weighting functions, and in [3] to allow the full assessment of the prospect theory utility function i.e. one that is defined over the whole domain of losses and gains. A number of authors [77, 105, 14] have reviewed generalizations to utility theory and developed procedures for the decomposition of multi-attribute non-expected utility functions, while others (e.g. [13] and [102]) relax the demands of probability theory by invoking concepts from Dempster-Shafer theory of evidence.

Unfortunately, these generalizations tend often to make the models even more complex and thus less transparent to decision makers, further aggravating difficulties of implementation. Our overall conclusion is thus that in the practical application of expected utility theory to decision making under uncertainty, the use of the additive aggregation model is likely to be adequate in a many settings. The imprecisions and uncertainties involved in constructing the partial utilities, which need in any case to be addressed by careful sensitivity analysis, are likely to outweigh any distinctions between the additive and multiplicative models. In fact, given that marginal utility functions based on preferences between hypothetical lotteries may generally not differ markedly from deterministic value functions based on relative strengths of preference (e.g. [100], Chapter 10), we conjecture that even the first step of the model construction could be based on the latter (e.g. by use of the SMART methodology, [100], Section 8.2). Some 
recent evidence in support of this view has been provided by [2]. Nevertheless, situations may arise when simplified utility models are simply inadequate, and some of the other models discussed below may need to be considered.

\section{Pairwise Comparisons}

As indicated in the previous section, the requirements of fitting a complete utility function can be extremely demanding both for the decision maker (in providing the necessary judgemental inputs) and for the analysts (in identifying complete multivariate distributions). We have seen how the assumption of a simple additive model may substantially reduce these demands without serious penalty in many practical situations. Nevertheless, other attempts at avoiding the construction of the full utility model have been made.

Even for single criterion models, the construction and validation of the complete utility model may be seen as too burdensome. Quite early work recognized, however, that it may often not be necessary to construct the full utility function in order to confirm whether one alternative is preferred to another. The conclusions may be derived from the concepts of stochastic dominance introduced by [47], and extended (to include third order stochastic dominance) by [99].

For purposes of defining stochastic dominance, suppose for the moment that there is only one criterion which we shall denote by $Z(x)$ (i.e. unsubscripted). Then let $P^{x}(z)$ be the (univariate) probability distribution function of $Z(x)$, i.e.: $P^{x}(z)=\operatorname{Pr}[Z(x) \leq z]$. With some abuse of notation, we shall use $P^{x}$ (without argument) to denote the probability distribution described by the function $P^{x}(z)$. Suppose also that values for $Z(x)$ are bounded between $z^{L}$ and $z^{U}$.

Three degrees of stochastic dominance may then be defined as follows.

First degree stochastic dominance $(F S D): P^{x}$ stochastically dominates $P^{y}$ in the first degree if and only $P^{x}(z) \leq P^{y}(z)$ for all $z \in\left[z^{L}, z^{U}\right]([47])$.

Second degree stochastic dominance $(S S D): P^{x}$ stochastically dominates $P^{y}$ in the second degree if and only:

$$
\int_{z^{L}}^{\zeta} P^{x}(z) d z \leq \int_{z^{L}}^{\zeta} P^{y}(z) d z
$$

for all $\zeta \in\left[z^{L}, z^{U}\right]([47])$.

Third degree stochastic dominance $(T S D): P^{x}$ stochastically dominates $P^{y}$ in the third degree if and only $\mathrm{E}[Z(x)] \geq \mathrm{E}[Z(y)]$ and:

$$
\int_{z^{L}}^{\eta} \int_{z^{L}}^{\zeta} P^{x}(z) d z d \zeta \leq \int_{z^{L}}^{\eta} \int_{z^{L}}^{z} P^{y}(z) d z d \zeta
$$

for all $\eta \in\left[z^{L}, z^{U}\right]([99])$. 
In this single-criterion case, the standard axioms of expected utility theory imply the existence of a utility function $u(z)$ such that $x \succ y$ if and only if:

$$
\int_{z^{L}}^{z^{U}} u(z) d P^{x}\left(z>\int_{z^{L}}^{z^{U}} u(z) d P^{y}(z) .\right.
$$

Without having explicitly to identify the utility function, however, considerations of stochastic dominance allow us to conclude the following ([8]):

1. If $P^{x}$ stochastically dominates $P^{y}$ in the first degree $\left(P^{x} F S D P^{y}\right)$, then $x \succ y$ provided that $u(z)$ is an increasing function of $z$ (which can be generally be assumed to be true in practical problems).

2. If $P^{x} S S D P^{y}$, then $x \succ y$ provided that $u(z)$ is a concave increasing function of $z$ (i.e. the decision maker is risk averse).

3. If $P^{x} T S D P^{y}$, then $x \succ y$ provided that $u(z)$ is a concave increasing function of $z$ with positive third derivative (corresponding to a risk averse decision maker exhibiting decreasing absolute risk aversion).

The potential importance of the above results lies in the claim which has been made that in practice some form of stochastic dominance may hold between many pairs of probability distributions. In other words, we may often be able to make pairwise comparisons between alternatives according to a particular criterion on the basis of stochastic dominance considerations, without needing to establish the partial value function for comparison of lotteries. In fact, we may often argue that FSD provides a strict pairwise preference, while SSD and TSD provide weaker forms of pairwise preference. Only in the absence of any stochastic dominance would we be unable to determine a preference without obtaining much stronger preference information from the decision maker.

Many of the more recent developments in this area have focussed on the problem of continuous optimization under stochastic dominance constraints (see, for example, [26]), often in the context of (single-criterion) portfolio optimization [83]. However, for discrete decision problems the existence of pairwise preferences at the level of a single criterion under uncertainty suggests that some form of outranking approach may be appropriate to aggregation across multiple criteria under uncertainty. A number of approaches [25, 72, 23, 32, 70] compare distributions by constructing a matrix $\mathbf{P}^{j}$ whose entries $P_{i k}^{j}$ denote the probability that alternative $a_{i}$ is superior to alternative $a_{k}$ on criterion $c_{j}$ i.e. $\operatorname{Pr}\left[Z_{i j} \geq Z_{k j}\right]$. The models differ with respect to the subsequent exploitation of the probabilities. [25] and [70] both aggregate the $P_{i k}^{j}$ using a weighted sum over attributes to arrive at a global index for each pairwise comparison $P_{i k}$. [32] compute joint probabilities associated with each of $2^{J}$ possible permutations of binary indicators denoting (attribute-specific) outranking between a pair of alternatives. Each of these is taken as evidence in favour of the 'superiority', 'inferiority', or 'indifference' of $a_{i}$ relative to $a_{k}$, based on a comparison with a user-defined threshold. A further algorithm is required to exploit the results. 
[72] incorporate more sophisticated outranking concepts such as indifference and preference thresholds, but subsequent aggregation and exploitation proceeds in a similar fashion to ELECTRE III. [23] compute stochastic "preference indices" measuring the degree of preference for one lottery over another in terms of one criterion, to be aggregated according to an outranking philosophy. Their preference indices may not be easily interpretable by many decision makers however, and perhaps with this problem in mind, [73] (but see also [5]) suggested an alternative outranking approach in which preferences according to individual criteria were established as far as possible by stochastic dominance considerations.

Martel and Zaras found it useful to introduce two forms of concordance index, which they term "explicable" and "non-explicable". For the "explicable" concordance, $x$ is judged at least as good as $y$ according to criterion $i$ if $P_{i}^{x}$ stochastically dominates $P_{i}^{y}$ at first, second or third degrees. This can be quite a strong assumption, as the preference assumption under TSD requires decreasing absolute risk aversion. The "non-explicable" concordance arises if neither of $P_{i}^{x}$ or $P_{i}^{y}$ stochastically dominates the other. The authors concede that in this case it is not certain that $x$ is at least as good as $y$, but they do combine the two indices under certain conditions. The discordance when comparing $x$ to $y$ is only non-zero in their model if $P_{i}^{y} F S D P_{i}^{x}$. The extensions of $[5,79,107]$ are largely concerned with constructing more fine-grained indices of stochastic dominance. Dominance-based methods have also been extended to make use of other data types, notably fuzzy numbers, and possibilistic and evidentiary evaluations $[106,12,19]$. These initially transform uncertain quantities so that they assume some of the properties of probability distributions before applying standard dominance concepts. Notably, this allows for the possibility of using several different data types in the same decision problem.

Although the implementation of many of the dominance-based approaches remain untested, they may have potential as an approach to dealing with uncertainty in MCDA using quite minimal preference information from the decision maker. This might at least be valuable for a first-pass screening of alternatives. Two problems may, however, limit wide applicability, especially in the MCDA context:

- Strong independence assumptions are implicitly made: The approach is based entirely on the marginal distributions of the elements of $\mathbf{Z}(x)$. This would only be valid if these elements (i.e. the criteria) were stochastically independent, or if the decision maker's preferences were additively independent in the sense of [56]. Either assumption would need to be carefully justified.

- Strong risk aversion assumptions are made: As indicated above, the method as proposed bases concordance measures on risk aversion and on decreasing absolute risk aversion. Especially the latter assumption may not always be easy to verify. The method can be weakened by basing concordance either only on FSD or on FSD and SSD, but this may not generate such useful results. 
There is clear scope for further research aimed at addressing the above problems.

\section{Risk Measures as Surrogate Criteria}

In this and the next sections, we move to more pragmatic approaches to dealing with uncertainty in the multicriteria context.

One obvious modelling approach is to view avoidance of risks as decision criteria in their own right. For example, the standard Markowitz portfolio theory (cf. [53]) represents a risky single-criterion objective (monetary reward) in terms of what are effectively two non-stochastic measures, namely expectation and standard deviation of returns. In this sense a single criterion decision problem under uncertainty is structured as a deterministic bi-criterion decision problem. The extension to risk components for each of number of fundamental criteria is obvious (see, for example, [75], p. 104, in the context of AHP).

There has, in fact, been a considerable literature on the topic of measuring risk for purposes of decision analysis, much of it motivated by the descriptive failures of expected utility theory. Papers by [86], [53], and by [62] contain many useful references. This literature is virtually entirely devoted to the single criterion case (typically financial returns), but it is worth recalling some of the key results with a view to extending the approaches to the multicriteria case.

The common theme has been that of developing axiomatic foundations for representation of psychological perceptions of risk (including consideration of importance and impact in addition to simple uncertainty), often based on some form of utility model. For example, [10] considers situations in which, if a decision maker switches from preferring one (typically more risky) lottery to another as his/her wealth increases, then he/she never switches back to preference for the first as wealth further increases. This he terms the "one-switch" rule for risk preferences, and demonstrates that if the decision maker is decreasingly risk averse, obeys the one switch rule, and approaches risk neutrality as total wealth tends to infinity, then the utility as a function of wealth $w$ must take on the form $w-b e^{-c w}$ for some positive parameters $b$ and $c$. Taking expectations results in an additive aggregation of two criteria, namely:

- The expectation of wealth (to be maximized); and

- The expectation of $b e^{-c w}$ (to be minimized), which can be viewed as a measure of risk.

[86] and [53] provide arguments for general moments of the distribution of returns (including but not restricted to variance) and/or expectations of terms such as $b e^{-c w}$, as measures of risk. While these may be useful as descriptive measures of risk behaviour, from the point of view of practical decision aid the use of variances to measure risk has been criticised for its symmetric treatment of gains are losses as well as its "ineffective" treatment of low-probability events [62]. It also seems doubtful whether a decision maker would be able to interpret anything but variance (or standard deviation) for purposes of providing 
necessary preference information (to establish tradeoffs, relative weights, goals, etc.).

More recent attention has focussed on a number of "downside" risk measures which consider only the impact of negative events. These include the semivariance $\mathrm{E}\left[(X-\mathrm{E}[X])^{2} \mid X<\mathrm{E}[X]\right]$, which measures the risk associated with obtaining a below-average performance and has been extended to an expected regret measure [24] using an arbitrary threshold $t$ rather than mean performance i.e. $\mathrm{E}\left[(X-t)^{2} \mid X<t\right]$. Two further measures of risk can be obtained by either defining an a priori desired probability level and assessing the associated quantile of performance (often referred to as 'variance-at-risk' in financial applications), or by defining an a priori target and assessing the probability of this target not being met. The use of quantiles (and, by extension, probabilities) for singleattribute risk modelling has been criticised, however, for (a) not accounting for extreme losses beyond the specified cut-off, (b) non-convexity, implying that the risk of a portfolio of alternatives may exceed the sum of the risks of its constituents, and (c) discontinuity with respect to the specified probability level [62]. The implications of these criticisms for MCDA have yet to be established, but it seems clear that the use of any more complex risk measures designed in response to these criticisms - in particular, 'conditional variance-at-risk' measuring expected losses conditional on losses exceeding a specified quantile - runs the risk of placing unrealistic demands on the decision maker's ability to assess inputs and interpret outputs. Limited empirical and simulation work which we have undertaken in the context of fisheries management ([90]) suggested that perceptions of risk of fishery collapse might be modelled better by probabilities of achieving one or more goals (in that case, periods of time before a collapse of the fishery). One advantage of such measures is that they might be much more easily interpreted by decision makers for purposes of expressing preferences or value judgements.

Given the apparent modelling success in representing preferences for single criterion problems under uncertainty by a simple additive aggregation of expected return and one or more risk measures (such as variance), there seems to be merit in exploring the extension of these results to the general multicriteria problem under uncertainty. In other words, each criterion (not necessarily financial) for which there exists substantial uncertainties might be restructured in terms of two separate criteria, viz. expected return and a measure of risk. Many of the above results produce an axiomatic justification for an additive aggregation of expected return and risk, so that these sub-criteria would be preferentially independent under the same axiomatic assumptions.

In spite of how obvious such multicriteria extensions might be, there seems to be little reference in the literature to explicit multicriteria modelling in which each criterion subject to uncertainty is decomposed into subcriteria representing expected return and risk. It is our experience, however, that various riskavoidance criteria arise almost naturally during the structuring phase of decision modelling, so that in practice risk avoidance criteria may in fact be more common than is apparent from the literature.

[58] has shown that evaluating alternatives by $\sum_{i=1}^{n}\left[w_{i} u_{i}\left(\mathrm{E}\left[Z_{i}\right]\right)-w_{i}^{R} \sigma_{i}^{2}\right]$ 
with "risk weights" defined by $w_{i}^{R}=(1 / 2) w_{i} u_{i}^{\prime \prime}\left(\mathrm{E}\left[Z_{i}\right]\right)$ can lead to close approximations of expected utility under the important conditions that the $Z_{i}$ be normally distributed and the underlying utility functions "do not deviate too much from linear". Other results [30], however, suggest that under strongly non-linear preferences this model can perform poorly.

Some of the few explicit references to multicriteria modelling in terms of a risk-return decomposition appear in the context of goal programming. For example, [6] expresses a stochastic multicriteria problem in terms of goals on combinations of risks and returns which are then solved by goal programming, but he does not separate out the risk and return components which may have led to a simpler model structure. [61] develops a multicriteria model for financial management, in which a number of different financial performance measures are used as criteria, some of which have a risk interpretation. Details of the solution procedure are not given, but the formulation clearly lends itself to a goal programming structure.

A somewhat earlier paper by [57] describes an integer goal programming model for capital budgeting, which can be viewed (together with the STRANGE method of [93]) as an extension of chance-constrained stochastic programming (see Elsevier volume for a broad introduction to stochastic programming). Keown and Taylor define goals in terms of desired probability levels, which may generically be expressed in the form:

$$
\operatorname{Pr}[g(Z) \leq \beta] \geq \alpha
$$

where $g(Z)$ is some performance function based on the unknown attribute values, $\beta$ the desired level of performance, and $\alpha$ a desired probability of achieving such performance. By using normal approximations, however, Keown and Taylor reduce the probability goal to one expressed in terms of a combination of mean and standard deviation which is subsequently treated in a standard goal programming manner. This suggests opportunity for research into investigation of generalized goal programming models which deal directly with deviations from both the desired performance levels ( $b$, above) and the desired probability levels ( $\alpha$, above).

Some work on fuzzy multiobjective programming (e.g. [22] and [21]) can be viewed in a similar manner, in the sense that a degree of anticipated level of goal achievement, measured in a fuzzy membership sense, may be interpreted as a risk measure.

Despite the attractiveness of using a single fixed target for each criteria, [20] show that this implies that an equivalent utility function formulation cannot be guaranteed. In order for such an equivalence to exist, the target must be probabilistic - an alternative formulation of the expected utility model is to assume a decision maker who has only two different utility levels depending on whether an uncertain target is met or not. The 'target-oriented' decision maker assesses probabilities $p(x)$ that the target is achieved given an attribute performance of $x$, rather than a utility function $u(x)$. [17] argue that in some circumstances this may be a "more intuitively appealing task", and extend the singleattribute results in [20] to show that for each multi-linear (or multiplicative or 
additive) utility function, there is an equivalent multi-linear (or multiplicative or additive) target-oriented formulation. In fact both the variance-based and probability-based goal programming models can be shown to be special cases of the target-oriented preference model [17].

More generally, the structuring of MCDA problems under uncertainty in terms of expected value and risk sub-criteria for each main criterion does have the advantage of being relatively simple and transparent to users. Such an approach appears to be easily integrated into any of the main MCDA methodologies, namely value measurement, outranking and goal programming/reference point methods. As indicated earlier, however, a decidedly open research question relates to the manner in which risk is most appropriately measured for this purpose.

A further practical issue is the extent to which the necessary independence properties can be be verified. In other words, to what extent can "risk" on one criterion be measured and assessed without taking into consideration ranges of uncertainties on the other criteria. Once again, this offers much scope for further research.

\section{Scenario Planning and MCDA}

Scenario planning ([95], but see also [35] for the decision support context) was developed as a technique for facilitating the process of identifying uncertain and uncontrollable factors which may impact on the consequences of decisions in the strategic management context. Scenario analysis has been widely accepted as an important component of strategic planning, and it is thus somewhat surprising how little appears to have been written concerning links between MCDA and scenario planning. A discussion of the link between scenario planning and decision making is provided by [48], but does not place this in an MCDA framework. Some multiobjective mathematical programming models, for example [69], do include some scenario concepts in an MCDM framework, but these scenarios tend to focus on technical and easily quantified components such as demands, rather than the richer "strategic conversation" espoused by van der Heiden. A broader review of the interrelationships between scenario planning and MCDA is given by [92].

One of the problems which arise in discussing scenarios is the lack of clear and agreed definitions of what is meant by a "scenario". [92] identified at least four distinctly different concepts which were summarized as follows:

Shell Scenario Planning Approach: This approach is well-documented by [95]. The emphasis is on constructing a coherent story of the future context against which the consequences of policies or strategies will be worked out. The intention of having alternative scenarios is primarily seen to be that of providing the basis for a "strategic conversation" concerning pros and cons of strategic decision options. The scenario relates to external events against which policies are compared and evaluated. It has been stressed in this approach that policy options do not form part of the scenario. 
Scenarios for exploring uncertainty: Scenarios may be used to explore how different uncertainties may play out, i.e. to explore a range of possible outcomes: see, e.g., [97]. In some senses this use of scenarios is similar to that within the Shell scenario planning context described above. The key difference is that there are no identified strategies needing to be evaluated against them. One simply explores possible futures, maybe to stimulate thinking about whether a change in strategy is necessary or whether there are opportunities that might be capitalized upon. Government Foresight studies are a good example of such a use: precursors to subsequent development and deliberation of specific strategies.

Scenarios for advocacy or political argument: This approach is allied to the previous two, but policy decisions or directions which are either being advocated or opposed are now explicitly integrated into the scenario, in order to emphasize plausible consequences of the policy directions. The purpose in producing the scenario is to create a story which highlights either the benefits or dangers of following one or other policy. [50] refers to utopian or dystopian perspectives being embedded in such uses of scenarios. The scenarios developed for South African political futures at a workshop involving a number of significant players during 1991/1992 are often held up as an example of this use of scenarios (and suggested as a major driver in the relatively peaceful transition which followed) ${ }^{1}$. Even the names chosen to describe the scenarios ("ostrich", "lame duck", "Icarus" and "flight of the flamingos") were chosen to evoke strong emotive responses. However significant these scenarios were in influencing the direction of negotiations in South Africa, they did not involve any analytical comparison of policy options ... the "flight of the flamingos" was embraced as self-evidently the only desirable future.

Representative sample of future states: This is a more technical approach. Future states are conceptualized in terms of a multivariate probability distribution on the state space. It is, however, recognized that the complete distribution may never be fully identified, and may in any case be too mathematically complicated to permit clear analysis of management options. For this reason, analysis will be based on a small number of representative outcomes in the sample space, but designed for good coverage as in experimental design, rather than selected randomly or because they seem "interesting".

The primary goal of scenario planning, at least in the first three perspectives above, is in the first instance to provide a structured "conversation" to sensitize decision makers to external and uncontrollable uncertainties, and to develop a shared understanding of such uncertainties. The approach is, however, naturally extended to the more analytical process of designing, evaluating and selecting

\footnotetext{
${ }^{1}$ For a detailed description, see Global Business Network, paper accessed on 4 Jan 2011 from http://www.generonconsulting.com/publications/papers/pdfs/Mont Fleur.pdf.
} 
courses of action on the basis of robustness to these uncertainties, which suggests close parallels with MCDA (as discussed, for example, by [39]). We shall explore these parallels shortly.

Scenarios are meant to represent fairly extreme futures than can still be viewed as plausible. As to what constitutes sufficiently "extreme" would depend on the facilitator, as in a very real sense, there will always be a possible future more extreme (and thus with greater potential impact on the consequences of decisions) than any which is incorporated into formal scenarios.

Van der Heijden suggests five principles which should guide scenario construction:

- At least two scenarios are required to reflect uncertainty, but more than four has proved (in his experience) to be impractical;

- Each scenario must be plausible, meaning that it can be seen to evolve in a logical manner from the past and present;

- Each scenario must be internally consistent;

- Scenarios must be relevant to the client's concerns and they must provide a useful, comprehensive and challenging framework against which the client can develop and test strategies and action plans;

- The scenarios must produce a novel perspective on the issues of concern to the client.

Once scenarios are constructed, they may be used to explore and to evaluate alternative strategies for the organization. Most proponents of scenario planning seem to avoid formal evaluation and analysis procedures, preferring to leave the selection of strategy to informed judgement. For example, [95] (pp. 232$235)$ rejects "traditional rationalistic decision analysis" as an approach which seeks to find a "right answer". This, however, represents are rather limited and technocratic perception of decision analysis, contrary to the constructive and learning view espoused by most in the MCDA field. The constructivist perspective is discussed at a number of places by [11] (see particularly Chapters 3,4 and 11), where it is argued that the underlying axioms are not meant to suggest a "right answer", but to provide a coherent discipline within which to construct preferences and strategies. Within such a view, the aims of scenario planning and MCDA share many commonalities, suggesting the potential for substantial synergies in seeking to integrate MCDA and scenario planning. On the one hand, MCDA can enrich the evaluation process in scenario planning, while the scenario planning approach can contribute to deeper understanding of the effects of external uncertainties in MCDA.

Various authors have hinted at the concept of scenarios in MCDA. These include, for example, [54]; [59], although this is largely in the context of a two state stochastic programming model; [98], also in a stochastic programming context; [75], Section 3, who refer to "states of nature"; [94] in the context of multiple objective linear programming; [76] and [69] in the context of power systems 
planning. These authors do not in general refer directly to the philosophical basis of scenario planning, however, and in some cases at least, the models are structured to suggest that the scenarios or states of nature constitute a complete sample space (see later).

[81] is one of the few to discuss scenario planning in the context of decision theory or decision analysis, but without substantive link to MCDA. He does however warn (page 199) of the danger that what might appear to be a robust choice of action (perhaps through unstructured and unsupported use of scenarios) may in fact be an illusion resulting from the fact that some events have simply been ignored. Such a danger suggests another perspective on the potential for two-way synergistic advantage between scenario planning and formal decision analysis: not only may scenario planning provide a means of dealing with uncertainties in MCDA, but decision analysis might contribute to avoiding of illusions of robustness or control in decision making. In the latter context, MCDA might contribute to the choice of scenarios as well as to the formal analysis of alternative courses of action.

Perhaps the closest formal link between MCDA and scenario planning is given in Chapter 14 of [39], which we sought to extend in [92]. In the remainder of this section, we outline these later extensions. Suppose that a set of $p$ scenarios indexed as $r=1,2, \ldots, p$ have been identified for purposes of evaluating alternatives. Let us then define $z_{i r}(x)$ (expressed by a lower case letter to emphasize that this is no longer viewed as a random variable) as the consequence of action $x$ in terms of criterion $i$, under the conditions defined by scenario $r$. As before, $\mathbf{z}_{r}(x)$ will represent the corresponding vector of consequences. We assume for each criterion $i$ and scenario $r$ that preferences are monotonically increasing with values of $z_{i r}(x)$, but we do not by any means imply that preferences are linear in the $\mathbf{z}_{r}(x)$. All that can be inferred is that an alternative $x$, say, is preferred to alternative $y$ (say) according to criterion $i$ under the assumptions of scenario $r$ if and only if $z_{i r}(x)>z_{i r}(y)$. If the scenarios are sufficiently rich to characterize the effects of uncertainties, then each alternative $x$ will to the same degree be sufficiently characterized by the 2-dimensional $(n \times p)$ array of performance measures $z_{i r}(x)$.

For the remainder of this section, we shall assume that the action space is finite, i.e. $X=\left\{x^{1}, x^{2}, \ldots, x^{q}\right\}$, say. For this case, [39] propose a three stage process based on a value function model:

1. Create an additive (multiattribute) value function model for the $n$ criteria, say $\sum_{i=1}^{n} w_{i} v_{i}\left(z_{i}\right)$, where the partial value functions $v_{i}\left(z_{i}\right)$ are defined over the range of $z_{i r}(x)$ values occurring across all scenarios.

2. For each alternative $x$ and scenario $r$, calculate $V_{r}(x)=\sum_{i=1}^{i} w_{i} v_{i}\left(z_{i r}(x)\right)$.

3. Display the $p \times q$ table of $V_{r}(x)$ values to the decision maker for a final selection, although Goodwin and Wright do not discuss modes of decision support for this final choice (implying that perceive it to be a relatively straightforward cognitive task, which we find difficult to accept in general). 
A critical assumption in the above approach is that of a scenario-independent value function, i.e. that value trade-offs between criteria are the same under all scenarios, which again we find far from self-evidently true in general. See for example [18] for a discussion on the dangers of assuming overly strong independence between scenarios.

[78] discuss practical problems which do arise in comparison of outcomes for all alternative-scenario combinations on a single basis. They proposed application of multiattribute value theory within each scenario, but accepting, for example, that weights associated with different criteria may, and quite typically do vary between scenarios. Their approach provides an evaluation of alternatives separately for each scenario, but they do not seek formal aggregation across scenarios. Rather, they seek to identify alternatives which are robust across scenarios in some sense.

The following example, which is a slight extension of that discussed in Section 3 , illustrates the difficulties in selecting between alternatives on the basis of the table of $V_{r}(x)$ values. In particular, it demonstrates that "robustness" across scenarios is not necessarily either well-defined or desirable when defined mainly in terms of variability in the $V_{r}(x)$ values.

Example: We have two alternatives $\left(x^{1}\right.$ and $\left.x^{2}\right)$, two criteria $\left(C_{1}\right.$ and $\left.C_{2}\right)$, two scenarios $\left(S_{1}\right.$ and $\left.S_{2}\right)$ and two possible outcomes (expressed as 0 or 1) on each criterion. Consequences for each action and scenario in terms of each criterion are given in Table 1.

Table 1: Description of consequences for the simple example

\begin{tabular}{lcclccc}
\hline Alternative & \multicolumn{2}{c}{ Scen. $S_{1}$} & & \multicolumn{2}{c}{ Scen. $S_{2}$} \\
\cline { 2 - 3 } \cline { 5 - 6 } & Crit. $C_{1}$ & Crit. $C_{2}$ & & Crit. $C_{1}$ & Crit. $C_{2}$ \\
\hline$x_{1}$ & 0 & 0 & & 1 & 1 \\
$x_{2}$ & 1 & 0 & & 0 & 1 \\
\hline
\end{tabular}

The important distinction between the two alternatives is that $x^{1}$ results in equal performance on both criteria under either scenario, while $x^{2}$ results in diametrically opposing performances on the two criteria under either scenario. As discussed in Section 3, there is no fundamental reason why one alternative should be preferred to the other. Concerns for equity between criteria would favour $x^{1}$, while an acceptance that good performance on one criterion might compensate for poorer outcomes on the other criterion might favour choice of $x^{2}$. A complete MAUT analysis would resolve the conflicts, but it is not clear that simpler aggregation methodologies would capture the relevant preferences. In the context of this example, any methodology should in its structure allow keep the door open to accept either $x^{1}$ or $x^{2}$ depending on the specific decision preferences which unfold. 
Without loss of generality, the marginal value functions for each of the two criteria can be defined such that $v_{i}(0)=0$ and $v_{i}(1)=1$ for both criteria. For the Goodwin-Wright approach, the $V_{r}(x)$ table becomes:

\begin{tabular}{lcc}
\hline Alternative & \multicolumn{2}{c}{ Scenarios } \\
\cline { 2 - 3 } & $S_{1}$ & $S_{2}$ \\
\hline$x^{1}$ & 0 & 1 \\
$x^{2}$ & $w_{1}$ & $w_{2}$ \\
\hline
\end{tabular}

This representation tends to obscure equity issues, and conventional robustness considerations seem likely to bias evaluation towards a form of risk aversion which would favour $x^{2}$.

There is a clear recognition that preference aggregation needs to be carried out across both the criteria and scenarios [39, 76, 69]. The view espoused by [92] is that in a scenario-based MCDA structure, alternatives do fundamentally need to be evaluated and compared in terms of all $p \times q$ performance measures identified earlier. In other words, at some point attention needs to be given to how well an alternative performs in terms of each criterion under the conditions of each scenario. In [92], we make this recognition explicit by reference to each criterion-scenario combination as a metacriterion. Each metacriterion represents a dimension on which preferences can and need to be formed and stated. In the above simple example, there are thus 4 metacriteria, corresponding to the last four columns of Table 1. Assuming that there is no alternative that is simultaneously best in terms of all $p \times q$ metacriteria, any decision made will reflect a balance between better performance on some metacriteria and lesser performance on others, i.e. there is an inevitable tradeoff between performances on each metacriterion, even if this may sometimes be difficult to express explicitly.

The scenario-based MCDA is thus equivalent to a standard multicriteria problem with $p \times q$ criteria (which we have termed metacriteria. In principle, any technique of MCDA could be applied to this metacriterion structure, but we illustrate the approach in terms of a value function methodology. Provided that the metacriteria are preferentially independent, standard results [e.g., 56, Chapter 5] imply that the alternatives may be ordered on the basis of an additive value function which can here be expressed in the form:

$$
V(x)=\sum_{i=1}^{n} \sum_{r=1}^{p} w_{i r} v_{i r}\left(z_{i r}(x)\right)
$$

where according to our structure, separate partial value functions need to be established for each criterion-scenario combination. This approach is illustrated below for our previous simple example.

Example (Continued). We can without loss of generality scale each marginal value function such that $v_{i r}(0)=0$ and $v_{i r}(1)=1$. Thus $V\left(x^{1}\right)=w_{12}+$ 
$w_{22}$ and $V\left(x^{2}\right)=w_{11}+w_{22}$, so that $x^{1}$ is preferred to $x^{2}$ if and only if $w_{12}>w_{11}$, and vice versa (with indifference if $w_{12}=w_{11}$ ).

Consider how the assessment of $w_{12}$ and $w_{11}$ might now proceed. We have that performance on criterion 2 is independent of action within each scenario, so that the performance on criterion 2 becomes a defining feature of the scenarios. The question to the decision maker is thus whether good performance on criterion 1 is more important in scenario 1 (characterized by poor outcomes on criterion 2 irrespective of action taken) or in scenario 2 (characterized by good outcomes on criterion 2). When inter-criterion compensation is beneficial, the first is more important; under concerns for equity, the second is more important. The necessity for such value judgements regarding compensation and equity concerns are clearly surfaced directly by the proposed methodology.

More generally, consider how metacriterion weights may be established. Swing-weighting is an established procedure for weight elicitation, but we need to recognize that the number of metacriteria will typically be too large to perform all swing-weighting comparisons simultaneously. Some form of hierarchical assessment may be needed, and two potential approaches may be recognized:

\section{Approach 1.}

- For each scenario $r$, compare the importance swings for each of the $n$ criteria within this scenario, giving estimates of the ratios $w_{i r} / w_{k r}$ for all pairs of criteria $i, k$;

- Then for one or two of the more important criteria, compare the relative importance of the swings for these criteria across each of the $p$ scenarios.

\section{Approach 2.}

- For each criterion $i$, compare the importance swings of criterion $i$ within each of the $p$ scenarios, giving estimates of the ratios $w_{i r} / w_{i s}$ for all pairs of scenarios $r, s$;

- Then for one or two selected scenarios, compare the relative importance of the swings for each of the $n$ criteria.

Neither approach differentiates in essence between the evaluation of importance of metacriteria within scenarios (comparisons of the initial criteria in a standard MCDA approach), or between scenarios (comparisons of scenarios). The distinction between the approaches is a matter of the timing of the comparisons during the analytical process. At this stage, we have not formed any clear conclusions as to which approach is preferable, which should form the topic of future empirical research. In the above simple example, however, either approach would recognize that $w_{2 r}=0$ for both scenarios (a zero swing having zero importance), leaving just the comparison of $w_{11}$ and $w_{12}$ to be undertaken, as indicated in the example (with the implied focus on importance of equity versus compensation). 


\section{Implications for Practice}

It should be evident from the preceding discussion that there still remains considerable scope for research into the treatment of substantive external uncertainties within an MCDA framework. It is hoped that such research will lead to ever-improved methodologies. Nevertheless, for the practitioner, certain guidelines can be given at the present time. These may be summarized as follows.

1. There is always a role for systematic sensitivity analysis for moderate levels of uncertainty, especially internal uncertainties, but care needs to be taken to avoid simple "one-at-a-time" variations in assumptions, as such an approach may miss interacting effects.

2. For those working within a value or utility function framework, the expectation of a simple additive value function can generate quite useful insights for the decision maker, provided that due attention is given to the shape (changing marginal values) of the function (cf. Stewart [88]). On the other hand, complete multiplicative or multilinear multiattribute utility functions may be difficult to implement correctly.

3. With any MCDA approach, there may be value and some theoretical justification in decomposing those criteria for which there is substantial uncertainty regarding outcomes, into two subcriteria of expected value and a risk measure respectively. An open question remains as to whether variance or standard deviation (which are conventionally used in this context) are the most appropriate risk measures for all problem types.

4. The integration of MCDA and scenario planning appears to be a potentially powerful tool, and may be particularly transparent to many decision makers. The approach is relevant to any methodology of MCDA. There do, nevertheless, remain some open questions, especially as regards the number of scenarios to be used and the means by which they are constructed or selected.

\section{References}

[1] Abdellaoui M (2000) Parameter-free elicitation of utility and probability weighting functions. Management Science pp 1497-1512

[2] Abdellaoui M, Barrios C, Wakker P (2007) Reconciling introspective utility with revealed preference: Experimental arguments based on prospect theory. Journal of Econometrics 138(1):356-378

[3] Abdellaoui M, Bleichrodt H, Paraschiv C (2007) Loss aversion under prospect theory: A parameter-free measurement. Management Science 53(10):1659-1674 
[4] Angilella S, Greco S, Matarazzo B (2010) Non-additive robust ordinal regression: A multiple criteria decision model based on the choquet integral. European Journal of Operational Research 201(1):277-288

[5] Azondékon SH, Martel JM (1999) "Value" of additional information in multicriterion analysis under uncertainty. European Journal of Operational Research 117:45-62

[6] Ballestero E (2001) Stochastic goal programming: A mean-variance approach. European Journal of Operational Research 131:476-481

[7] Banuelas R, Antony J (2007) Application of stochastic analytic hierarchy process within a domestic appliance manufacturer. Journal of the Operational Research Society 58(1):29

[8] Bawa VS (1975) Optimal rules for ordering uncertain prospects. Journal of Financial Economics 2:95-121

[9] Bazerman MH (2002) Judgment in Managerial Decision Making, 5th edn. John Wiley \& Sons, New York

[10] Bell DE (1988) One-switch utility functions and a measure of risk. Management Science 34:1416-1424

[11] Belton V, Stewart TJ (2002) Multiple Criteria Decision Analysis: An Integrated Approach. Kluwer Academic Publishers, Boston

[12] Ben Amor S, Jabeur K, Martel J (2007) Multiple criteria aggregation procedure for mixed evaluations. European journal of operational research 181(3):1506-1515

[13] Beynon M, Curry B, Morgan P (2000) The Dempster-Shafer theory of evidence: an alternative approach to multicriteria decision modelling. OMEGA: International Journal of Management Science 28:37-50

[14] Bleichrodt H, Miyamoto J (2003) A characterization of quality-adjusted life-years under cumulative prospect theory. Mathematics of Operations Research pp 181-193

[15] Bleichrodt H, Pinto J (2000) A parameter-free elicitation of the probability weighting function in medical decision analysis. Management Science pp $1485-1496$

[16] Bleichrodt H, Pinto J, Wakker P (2001) Making descriptive use of prospect theory to improve the prescriptive use of expected utility. Management Science pp 1498-1514

[17] Bordley R, Kirkwood C (2004) Multiattribute Preference Analysis with Performance Targets. Operations Research 52(6):823 
[18] Bordley RF, Hazen G (1992) Nonlinear utility models arising from unmodelled small world intercorrelations. Management Science 38:1010-1017

[19] Boujelben M, Smet Y, Frikha A, Chabchoub H (2009) Building a binary outranking relation in uncertain, imprecise and multi-experts contexts: The application of evidence theory. International journal of approximate reasoning 50(8):1259-1278

[20] Castagnoli E, Calzi M (1996) Expected utility without utility. Theory and Decision 41(3):281-301

[21] Chang NB, Wang S (1997) A fuzzy goal programming approach for the optimal planning of metropolitan solid waste management systems. European Journal of Operational Research 99:303-321

[22] Chang NB, Wen C, Chen Y (1997) A fuzzy multi-objective programming approach for optimal management of the reservoir watershed. European Journal of Operational Research 99:289-302

[23] D'Avignon GR, Vincke P (1988) An outranking method under uncertainty. European Journal of Operational Research 36:311-321

[24] Dembo R, Rosen D (1999) The practice of portfolio replication. a practical overview of forward and inverse problems. Annals of Operations Research $85: 267-284$

[25] Dendrou B, Dendrou S, Houstis E (1980) Multiobjective decision analysis for engineering systems. Computers and Operations Research 7:301-312

[26] Dentcheva D, Ruszczynski A (2003) Optimization with stochastic dominance constraints. SIAM Journal on Optimization 14(2):548-566

[27] Dimitras A, Slowinski R, Susmaga R, Zopounidis C (1999) Business failure prediction using rough sets. European Journal of Operational Research 114:263-280

[28] Durbach I (2006) A simulation-based test of stochastic multicriteria acceptability analysis using achievement functions. European Journal of Operational Research 170:923-934

[29] Durbach I, Davis S (2012) Decision support for selecting a shortlist of electricity-saving options: A modified SMAA approach. ORiON 28(2):99116

[30] Durbach I, Stewart T (2009) Using expected values to simplify decision making under uncertainty. Omega 37(2):312-330

[31] Durbach IN, Stewart TJ (2012) Modelling uncertainty in multi-criteria decision analysis. European Journal of Operational Research 223:1-14 
[32] Fan Z, Liu Y, Feng B (2010) A method for stochastic multiple criteria decision making based on pairwise comparisons of alternatives with random evaluations. European Journal of Operational Research 207(2):906-915

[33] Fishburn PC (1984) Foundations of risk measurement. I. Risk as probable loss. Management Science 30:396-406

[34] French S (1995) Uncertainty and imprecision: modelling and analysis. Journal of the Operational Research Society 46:70-79

[35] French S, Maule J, Papamichail N (2009) Decision Behaviour, Analysis and Support. Cambridge University Press, Campbridge

[36] Friend J (2001) The strategic choice approach. In: Rosenhead J, Mingers J (eds) Rational Analysis for a Problematic World Revisited, 2nd edn, John Wiley \& Sons, Chichester, pp 115-149

[37] Gal T, Stewart TJ, Hanne T (eds) (1999) Multicriteria Decision Making: Advances in MCDM Models, Algorithms, Theory, and Applications. Kluwer Academic Publishers, Boston

[38] Goicoechea A, Hansen DR, Duckstein L (1982) Multiobjective Decision Analysis with Engineering and Business Applications. John Wiley \& Sons, New York

[39] Goodwin P, Wright G (2009) Decision Analysis for Management Judgement, 4th edn. John Wiley and Sons, Chichester

[40] Greco S, Matarazzo B, Slowinski R (1999) Rough approximation of a preference relation by dominance relations. European Journal of Operational Research 117:63-83

[41] Greco S, Matarazzo B, Slowinski R (1999) The use of rough sets and fuzzy sets in MCDM. In: [37], chap 14

[42] Greco S, Matarazzo B, Slowinski R (2001) Rough sets theory for multicriteria decision analysis. European Journal of Operational Research 129:147

[43] Greco S, Matarazzo B, Slowinski R (2002) Rough sets methodology for sorting problems in presence of multiple attributes and criteria. European Journal of Operational Research 138:247259

[44] Greco S, Mousseau V, Słowiński R (2010) Multiple criteria sorting with a set of additive value functions. European Journal of Operational Research 207(3):1455-1470

[45] Greco S, Słowiński R, Figueira J, Mousseau V (2010) Robust ordinal regression. In: Greco S, Ehrgott M, Figuera J (eds) Trends in Multiple Criteria Decision Analysis, Springer, chap 9 
[46] Greco S, Kadziński M, Mousseau V, Słowiński R (2011) ELECTREGKMS: Robust ordinal regression for outranking methods. European Journal of Operational Research 214(1):118-135

[47] Hadar J, Russell WR (1969) Rules for ordering uncertain prospects. The American Economic Review 59:25-34

[48] Harries C (2003) Correspondence to what? Coherence to what? What is good scenario-based decision making. Technological Forecasting \& Social Change 70:797-817

[49] Hokkanen J, Lahdelma R, Miettinen K, Salminen P (1998) Determining the implementation order of a general plan by using a multicriteria method. Journal of Multi-Criteria Decision Analysis 7(5):273-284

[50] Hughes N (2009) A historical overview of strategic scenario planning. Tech. rep., UKERC and EON.UK/EPSRC Project on Transition Pathways to a Low Carbon Economy

[51] Insua D, French S (1991) A framework for sensitivity analysis in discrete multi-objective decision-making. European Journal of Operational Research 54(2):176-190

[52] Jacquet-Lagrèze E, Siskos Y (2001) Preference disaggregation: 20 years of MCDA experience. European Journal of Operational Research 130:233245

[53] Jia J, Dyer JS (1996) A standard measure of risk and risk-value models. Management Science 42:1691-1705

[54] Jiménez A, Mateos A, Ros-Insua S (2009) Missing consequences in multiattribute utility theory. Omega: The International Journal of Management Science 37:395-410

[55] Kahneman D, Tversky A (1979) Prospect theory: An analysis of decision under risk. Econometrica 47:263-291

[56] Keeney RL, Raiffa H (1976) Decisions with Multiple Objectives. J. Wiley $\&$ Sons, New York

[57] Keown AJ, Taylor III BW (1980) A chance-constrained integer goal programming model for capital budgeting in the production area. Journal of the Operational Research Society 31:579-589

[58] Kirkwood C (1992) Estimating the impact of uncertainty on deterministic multiattribute evaluation. Management Science 38(6):819-826

[59] Klein G, Moskowitz H, Ravindran A (1990) Interactive multiobjective optimization under uncertainty. Management Science 36:58-75 
[60] Klir GJ, Folger TA (1988) Fuzzy Sets, Uncertainty and Information. Prentice Hall, Englewood Cliffs, New Jersey

[61] Korhonen A (2001) Strategic financial management in a multinational financial conglomerate: A multiple goal stochastic programming approach. European Journal of Operational Research 128:418-434

[62] Krokhmal P, Zabarankin M, Uryasev S (2011) Modeling and optimization of risk. Surveys in Operations Research and Management Science 16(2):49-66

[63] Lahdelma R, Salminen P (2001) SMAA-2: stochastic multi-criteria acceptability analysis for group decision making. Operations Research 49(3):444-454

[64] Lahdelma R, Salminen P (2009) Prospect theory and stochastic multicriteria acceptability analysis (SMAA). Omega 37(5):961-971

[65] Lahdelma R, Hokkanen J, Salminen P (1998) SMAA - Stochastic multiobjective acceptability analysis. European Journal of Operational Research 106:137-143

[66] Lahdelma R, Miettinen K, Salminen P (2005) Reference point approach for multiple decision makers. European Journal of Operational Research 164(3):785-791

[67] Levary R, Wan K (1999) An analytic hierarchy process based simulation model for entry mode decision regarding foreign direct investment. Omega 27(6):661-677

[68] Levary RR, Wan K (1998) A simulation approach for handling uncertainty in the analytic hierarchy process. European Journal of Operational Research 106:116-122

[69] Linares P (2002) Multiple criteria decision making and risk analysis as risk management tools for power systems planning. IEEE Transactions on Power Systems 17:895-900

[70] Liu Y, Fan Z, Zhang Y (2011) A method for stochastic multiple criteria decision making based on dominance degrees. Information Sciences 181(19):4139-4153

[71] Mareschal B (1986) Stochastic multicriteria decision making and uncertainty. European Journal of Operational Research 26:58-64

[72] Martel J, d'Avignon G, Couillard G (1986) A fuzzy outranking relation in multicriteria decision making. European Journal of Operational Research $25: 258-271$

[73] Martel JM, Zaras K (1995) Stochastic dominance in multicriterion analysis under risk. Theory and Decision 39:31-49 
[74] Mateos A, Ríos-Insua S, Jiménez A (2006) Dominance, potential optimality and alternative ranking in imprecise multi-attribute decision making. Journal of the Operational Research Society 58(3):326-336

[75] Millet I, Wedley WC (2002) Modelling risk and uncertainty with the Analytic Hierarchy Process. Journal of Multi-Criteria Decision Analysis 11:97107

[76] Miranda V, Proença LM (1998) Why risk analysis outperforms probabilistic choice as the effective decision support paradigm for power system planning. IEEE Transactions on Power Systems 13:643-648

[77] Miyamoto JM, Wakker P (1996) Multiattribute utility theory without expected utility foundations. Operations Research 44:313-326

[78] Montibeller G, Gummer H, Tumidei D (2006) Combining scenario planning and multi-criteria decision analysis in practice. Journal of MultiCriteria Decision Analysis 14:5-20

[79] Nowak M (2004) Preference and veto thresholds in multicriteria analysis based on stochastic dominance. European Journal of Operational Research 158(2):339-350

[80] Parnell GS, Jackson JA, Burk RC, Lehmkuhld LJ, Engelbrecht Jr JA (1999) R\&D concept decision analysis: using alternate futures for sensitivity analysis. Journal of Multi-Criteria Decision Analysis 8:119-127

[81] Pomerol JC (2001) Scenario development and practical decision making under uncertainty. Decision Support Systems 31:197-204

[82] Rios Insua D (1990) Sensitivity Analysis in Multi-Objective Decision Making. Springer (Lecture Notes in Economics and Mathematical Systems, Vol. 347), Berlin

[83] Roman D, Darby-Dowman K, Mitra G (2006) Portfolio construction based on stochastic dominance and target return distributions. Mathematical Programming 108(2):541-569

[84] Rosqvist T (2001) Simulation and multi-attribute utility modelling of life cycle profit. Journal of Multi-Criteria Decision Analysis 10:205-218

[85] Saltelli A, Tarantola AS, Chan K (1999) A role for sensitivity analysis in presenting the results from MCDA studies to decision makers. Journal of Multi-Criteria Decision Analysis 8:139-145

[86] Sarin RK, Weber M (1993) Risk-value models. European Journal of Operational Research 70:135-149

[87] Shafer G (1976) A Mathematical Theory of Evidence. Princeton University Press, Princeton 
[88] Stewart TJ (1995) Simplified approaches for multi-criteria decision making under uncertainty. Journal of Multi-Criteria Decision Analysis 4:246-258

[89] Stewart TJ (1996) Robustness of additive value function methods in MCDM. Journal of Multi-Criteria Decision Analysis 5:301-309

[90] Stewart TJ (1998) Measurements of risk in fisheries management. ORiON 14:1-15

[91] Stewart TJ (1999) Concepts of interactive programming. In: [37], chap 10

[92] Stewart TJ, French S, Rios J (2013) Integrating multicriteria decision analysis and scenario planning - review and extension. Omega, The International Journal of Management Science 41:679-688, DOI 10.1016/j. omega.2012.09.003, dOI: 10.1016/j.omega.2012.09.003 (To appear)

[93] Teghem Jr J, Dufrane D, Thauvoye M (1986) STRANGE: An interactive method for multi-objective linear programming under uncertainty. European Journal of Operational Research 26:65-82

[94] Urli B, Nadeau R (2004) PROMISE/scenarios: An interactive method for multiobjective stochastic linear programming under partial uncertainty. European Journal of Operational Research 155:361-372

[95] Van der Heijden K (1996) Scenarios: The Art of Strategic Conversation. John Wiley \& Sons, Chichester

[96] Wakker P, Deneffe D (1996) Eliciting von Neumann-Morgenstern utilities when probabilities are distorted or unknown. Management Science pp $1131-1150$

[97] Walker WE, Harremoes P, Rotmans J, van der Sluijs JP, van Asselt MBA, Janssen P, Krayer von Krausswalker MP (2003) Defining uncertainty: a conceptual basis for uncertainty management in model based decision support. Integrated Asessment 4(1):5-17

[98] Watkins Jr DW, McKinney DC, Lasdon LS, Nielsen SS, Martin QW (2000) A scenario-based stochastic programming model for water supplies from the highland lakes. International Transactions in Operational Research 7:211-230

[99] Whitmore GA (1970) Third order stochastic dominance. The American Economic Review 60:457-459

[100] von Winterfeldt D, Edwards W (1986) Decision Analysis and Behavioral Research. Cambridge University Press, Cambridge

[101] Xu D (2012) An introduction and survey of the evidential reasoning approach for multiple criteria decision analysis. Annals of Operations Research 195(1):163-187 
[102] Yang JB (2001) Rule and utility based evidential reasoning approach for multiattribute decision analysis under uncertainties. European Journal of Operational Research 131:31-61

[103] Yeh C, Deng H, Pan H (1999) Multi-criteria analysis for dredger dispatching under uncertainty. Journal of the Operational Research Society $50: 35-43$

[104] Yilmaz MR (1992) An information-expectation framework for decisions under uncertainty. Journal of Multi-Criteria Decision Analysis 1:65-80

[105] Zank H (2001) Cumulative prospect theory for parametric and multiattribute utilities. Mathematics of Operations Research pp 67-81

[106] Zaras K (2004) Rough approximation of a preference relation by a multiattribute dominance for deterministic, stochastic and fuzzy decision problems. European Journal of Operational Research 159(1):196-206

[107] Zhang Y, Fan Z, Liu Y (2010) A method based on stochastic dominance degrees for stochastic multiple criteria decision making. Computers \& Industrial Engineering 58(4):544-552

[108] Zimmermann H (2000) An application-oriented view of modeling uncertainty. European Journal of Operational Research 122:190-198 